\title{
Analyzing the Burden of Revision Total Knee Arthroplasty in the United States between 2009 and 2016
}

Isaac Livshetz, MD ${ }^{1}$ Benjamin H. Sussman, BS ${ }^{2}$ Vivian Papas, MS ${ }^{3}$ Nequesha S. Mohamed, MD Hytham S. Salem, MD ${ }^{3}$ Ronald E. Delanois, MD ${ }^{4}$ Michael A. Mont, MD ${ }^{3}$ Giles R. Scuderi, MD ${ }^{3}$

${ }^{1}$ Department of Orthopedic Surgery, White Plains Hospital, White Plains, New York

${ }^{2}$ Department of Orthopedic Surgery, Lewis Katz School of Medicine, Temple University, Philadelphia, Pennsylvania

${ }^{3}$ Department of Orthopedic Surgery, Lenox Hill Hospital Northwell Health, New York, New York

${ }^{4}$ Department of Orthopedic Surgery, Center for Joint Preservation and Replacement, Rubin Institute for Advanced Orthopedics, Sinai Hospital of Baltimore, Baltimore, Maryland

J Knee Surg 2023;36:121-131.

\author{
Address for correspondence Michael A. Mont, MD, Lenox Hill \\ Hospital, 130 East 77th Street, 11th Floor, New York, NY 10075 \\ (e-mail: rhondamont@aol.com).
}

\begin{abstract}
Keywords

- knee

- total knee arthroplasty

- revision

- economics

As the number of total knee arthroplasties (TKAs) increases, it is reasonable to expect the number of revision TKAs (rTKAs) to rise in parallel. The patient-related and societal burdens of rTKA are poorly understood. Therefore, the purpose of this study was to determine temporal changes in: (1) the incidence of rTKA; (2) patient and hospital characteristics; (3) complications, hospital lengths of stay (LOSs), and discharge dispositions; and (4) costs, charges, and payer types. All patients who underwent rTKA between 2009 and 2016 were identified from the National Inpatient Sample database using International Classification of Diseases, Ninth Revision and Tenth Revision codes and were studied. Univariate analyses were performed to compare the incidence of rTKA, patient and hospital characteristics, LOS and discharge dispositions, as well as costs, charges, and payer types. A multivariate logistic regression model was built to compare the odds of complications in 2009 and 2016. Over our study period, there was a $4.3 \%$ decrease in the incidence of rTKA. The mean age of patients who underwent rTKA was 65 years and a majority were female (58\%). Mean hospital LOS decreased from 4.1 days in 2009 to 3.3 days in 2016 $(p<0.001)$. The rate of several complications decreased significantly over our study period including myocardial infarction, cardiac arrest, transfusion, pneumonia, urinary tract infection, and mortality. A significantly lower percentage of rTKA patients were discharged to a skilled nursing facility in 2016 (26.5\%) compared with 2009 (31.6\%; $p<0.001)$. There was an $18.7 \%$ increase in the mean costs, and a $43.3 \%$ increase in the mean charges $(p<0.001)$. Over the study period, there was a decrease in the incidence of rTKAs. Despite potential improvements in primary TKA, the burden associated with rTKA remains large. This report can be used to help educate medical providers about outcomes that may result from a primary and/or revised TKA.
\end{abstract}

received

July 13,2020

accepted after revision

May 1, 2021

article published online

July 8,2021 (c) 2021. Thieme. All rights reserved.

Thieme Medical Publishers, Inc., 333 Seventh Avenue, 18th Floor, New York, NY 10001, USA
DOI https://doi.org/

10.1055/s-0041-1731324.

ISSN 1538-8506. 
Despite the continued success of primary total knee arthroplasty (TKA), the incidence of revision surgery is increasing. Regardless of the cause, revision surgery poses a large burden for patients, surgeons, and the health care system..$^{1-5}$ Recent literature has reported that the predominant reasons for revisionTKA (rTKA) include aseptic loosening, periprosthetic joint infection, polyethylene wear, and instability. ${ }^{1,6-10}$ However, it has been shown that the causes for rTKA have changed over time.

The economic burden of rTKA is substantial, and postoperative hospital lengths of stay (LOSs) are suggested to be a major contributor of incurred costs. ${ }^{11,12}$ Despite decreasing postoperative LOSs among patients who undergo rTKA, hospital charges associated with these procedures continue to increase. ${ }^{1,4,13-15}$ To address the economic issues associated with rTKA, it is important to maintain a good understanding of the epidemiologic trends associated with this procedure.

The purpose of this study was to examine the economic burden, incidence, complications, LOSs, charges, and costs of rTKAs on the U.S. health care system between 2009 and 2016. Our primary goals were to determine: (1) the temporal changes in rTKAs patient demographics, postoperative hospital LOS, inpatient complications, discharge disposition, payer types, charges, and costs; (2) the incidence of rTKAs across all age groups over time; (3) trends in the most prevalent diagnoses leading to rTKAs; and (4) the temporal changes in hospital characteristics including hospital type and hospital bed size.

\section{Materials and Methods}

\section{Database Information}

The database utilized was the National Inpatient Sample (NIS) from the Healthcare Cost and Utilization Project
(HCUP) (Agency of Healthcare Research Quality, Rockville, MD). ${ }^{16}$ The NIS, which is the largest all-payer national database, comprised $20 \%$ sample of all national hospital inpatient stays. ${ }^{17}$ This database represents more than 8 million annual discharges in the United States. Due to the public availability and deidentified data from the NIS, this study was exempted from our institution's Institutional Review Board approval.

\section{Patient Selection}

The NIS was searched using the International Classification of Diseases, Ninth Revision (ICD-9) codes to identify all patients who underwent rTKAs between January 1, 2009, and December 31,2016 . The codes that were utilized were 00.80 , $00.81,00.82,00.83,00.84$, and 81.55. A total of 482,851 patients were included for analysis (-Fig. 1). Further stratification by year yielded 51,670 patients in 2009 and 49,430 patients in 2016.

\section{Patient and Hospital Characteristics}

The rTKAs rate was calculated based on the yearly U.S. population. We initially divided the incidence of rTKAs (derived from the NIS) by the U.S. census estimates, and then multiplied the result by 100,000. The following variables were collected for all patients: main causes for rTKAs, age groups (younger than 44 years, 45-64 years, 65-85 years, older than 86 years), sex, severity of injury (SOI) (minor, moderate, major, and severe), LOS, discharges to skilled nursing facilities (SNFs), payers (i.e., Medicare, Medicaid, private insurance, self-pay, and other), charges, and costs. ${ }^{18}$ Resource costs are the actual expenses required to provide the services. ${ }^{19}$ Examples of costs include, but are not limited

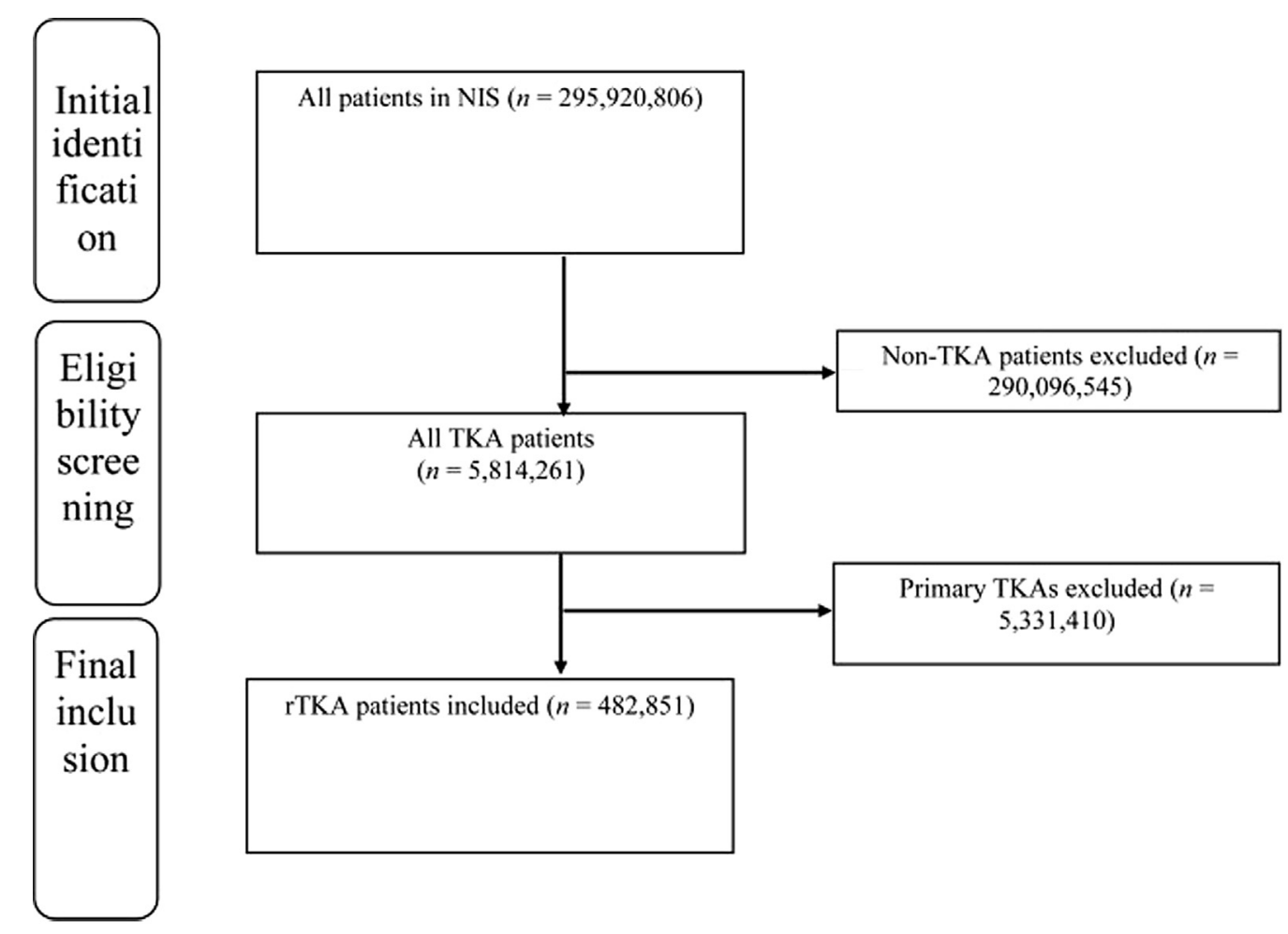

Fig. 1 Flowchart for study inclusion. rTKA, revision total knee arthroplasty; TKA, total knee arthroplasty. 
Table 1 ICD codes used to identify implant-related complications

\begin{tabular}{|l|l|}
\hline ICD-9 code & Description \\
\hline 996.41 & Mechanical loosening of prosthetic joint \\
\hline 996.42 & Dislocation of prosthetic joint \\
\hline 996.43 & Prosthetic joint implant failure/breakage \\
\hline 996.44 & $\begin{array}{l}\text { Periprosthetic fracture around prosthetic } \\
\text { joint }\end{array}$ \\
\hline 996.45 & Periprosthetic osteolysis \\
\hline 996.46 & $\begin{array}{l}\text { Articular bearing surface wear of prosthetic } \\
\text { joint }\end{array}$ \\
\hline 996.47 & $\begin{array}{l}\text { Other mechanical complication of pros- } \\
\text { thetic joint implant }\end{array}$ \\
\hline 996.49 & $\begin{array}{l}\text { Other mechanical complication of other } \\
\text { internal orthopedic device, implant, or } \\
\text { graft }\end{array}$ \\
\hline 996.66 & $\begin{array}{l}\text { Infection and inflammatory reaction due to } \\
\text { internal joint prosthesis }\end{array}$ \\
\hline 996.77 & $\begin{array}{l}\text { Other complications due to internal joint } \\
\text { prosthesis }\end{array}$ \\
\hline
\end{tabular}

Abbreviation: ICD, International Classification of Diseases.

to, supplies, utility, and wages. ${ }^{19}$ Cost data were calculated using the supplemental cost-to-charge ratio files provided by the HCUP for analysis, and adjusted to 2019 dollars using the consumer price index. ${ }^{19}$ The ICD codes listed in -Table 1 were used to identify implant-related complications. Inpatient complications were also identified by ICD codes and included myocardial infarctions, pulmonary emboli/deep vein thromboses, cardiac arrests, transfusions, pneumoniae, urinary tract infections, and deaths. The hospital variables collected included hospital types (e.g., urban nonteaching, urban teaching, and rural) and hospital sizes determined by the number of beds (e.g., small, medium, and large). This study also temporally compared amount of rTKAs, age, sex, inpatient complications, reasons for rTKAs, SOI, payer types, hospital types, and hospital sizes between 2009 and 2016.

\section{Statistical Analyses}

All statistical analyses were conducted using SPSS version 25 (IBM Corporation, Armonk, NY). Tables and figures were generated via Excel (Microsoft Corporation, Redmond, WA). Results for categorical variables were presented as frequencies and percentages, while those for continuous variables were presented as means and standard deviations. Descriptive statistics were reported for each year between 2009 and 2016 to provide a snapshot of patient demographics and trends of all of the studied variables for the duration of the study. For all studied variables (incidence of rTKAs, diagnoses leading to rTKAs, postoperative complication rates, patient demographics, hospital and payer types, LOS, discharge dispositions, charges, and costs), data from the year 2009 were compared with those from 2016. Chi-square analyses were performed to compare categorical variables (sex, SOI, payer type, hospital type, and hospital bed size), in 2009 and 2016. Independent sample $t$-tests were used to compare continuous variables (age, LOS, charges, and costs) in 2009 and 2016. Multivariate logistic regression models were used to compare the rate of inpatient complications following rTKAs (e.g., myocardial infarction, pulmonary embolism/deep vein thrombosis, cardiac arrest, transfusion, pneumonia, urinary tract infection, discharge to SNF, and death) in 2009 and 2016 while controlling for patient age, sex, and SOI. Results of the logistic regression analyses were presented as odds ratios (ORs) and 95\% confidence intervals (CIs). Statistically significant results from the logistic regression model were determined by $95 \% \mathrm{CIs}$ that did not include the null value $(=1)$, while those from all other analyses were indicated by $p$-values less than 0.05 .

\section{Results}

\section{Incidence of Revision TKAs}

A total of 453,770 rTKAs were performed between 2009 and 2016. The overall incidence decreased by $4.34 \%$; from 51,670 in 2009 to 49,430 in 2016 (-Table 2, - Fig. 2). Age-adjusted population rate per 100,000 persons demonstrated a $9.25 \%$ decreased incidence of revision across all age groups (-Table 3). The population-adjusted incidence decreased $30.4 \%$ in the $\leq 44$ years age group, $5.73 \%$ in the 45 to 64 years age group, $9.4 \%$ in the 65 to 85 years age group, and $28.3 \%$ in the $\geq 85$ years age group. The incidence of rTKAs was lowest in the oldest and youngest age groups, and highest in patients aged 45 to 85 years (-Fig. $\mathbf{3}$ ).

The four most prevalent diagnoses that led to rTKAs in 2009 were mechanical loosenings (19.7\%), infections (14.7\%), other complications due to internal joint prostheses (12.4\%), and broken prosthetic joint implants (12.2\%). Over time, the leading causes for rTKAs changed. In 2016, mechanical

Table 2 Nationwide incidence of TKA revisions in the United States (2009-2016)

\begin{tabular}{|l|l|l|l|l|l|l|l|l|l|}
\hline TKA revisions & $\mathbf{2 0 0 9}$ & $\mathbf{2 0 1 0}$ & $\mathbf{2 0 1 1}$ & $\mathbf{2 0 1 2}$ & $\mathbf{2 0 1 3}$ & $\mathbf{2 0 1 4}$ & $\mathbf{2 0 1 5}$ & $\mathbf{2 0 1 6}$ & \% change \\
\hline All ages & 51,670 & 58,431 & 61,969 & 58,930 & 60,315 & 62,560 & 50,465 & 49,430 & -4.34 \\
\hline Age $\leq 44$ y & 1,690 & 2,099 & 2,256 & 1,890 & 1,935 & 1,925 & 1,240 & 1,240 & -26.33 \\
\hline Age $45-64$ y & 22,139 & 25,997 & 27,896 & 26,445 & 26,445 & 27,480 & 21,575 & 22,000 & -0.63 \\
\hline Age $65-85$ y & 25,823 & 28,172 & 29,606 & 28,650 & 29,920 & 30,990 & 25,740 & 24,665 & -4.48 \\
\hline Age $\geq 85$ y & 2,018 & 2,163 & 2,211 & 1,945 & 2,015 & 2,165 & 1,910 & 1,525 & -24.43 \\
\hline
\end{tabular}

Abbreviation: TKA, total knee arthroplasty. 


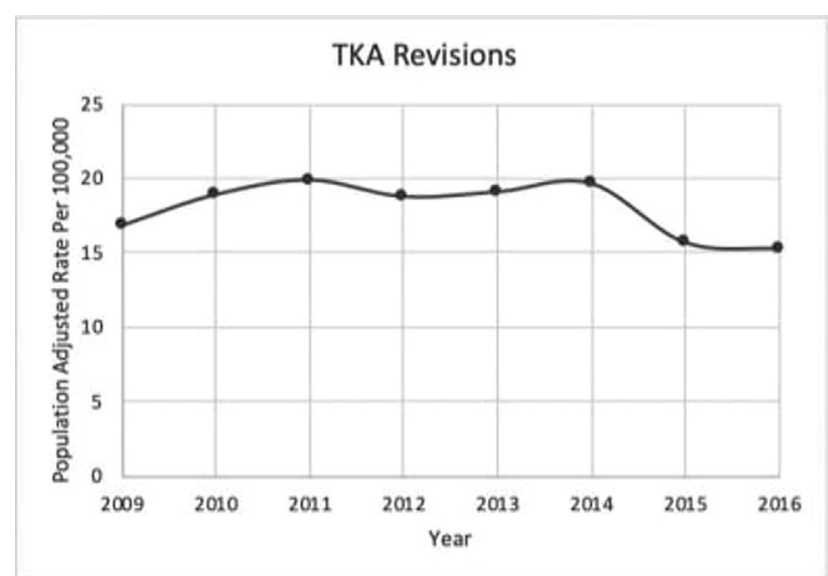

Fig. 2 Nationwide Incidence of total knee arthroplasty (TKA) revisions in the United States between 2009 and 2016.

loosenings remained the most common reason for revision (28.4\%). Other mechanical complications of internal prostheses (12.8\%) became the second most common diagnosis, while infections (12.2\%) were the third leading cause of rTKAs. The fourth most common diagnosis that led to rTKAs changed to instabilities (10.7\%). The fourth most common reason in 2009 (broken prosthetic joints [12.2\%]) became the ninth most common reason for revision in 2016 (1.3\%; - Table 4).

\section{Patient and Hospital Characteristics}

Mean age at the time of rTKAs was $\sim 65$ years in 2009 and 2016 (-Table 5). Female patients comprised 57.9\% of our sample population in 2009 compared with 58.4\% in 2016 (-Table 6). There were significant changes in the distribution of SOI scores between 2009 and 2016 ( $p<0.001$; - Table 6). The size and type of hospitals in which rTKAs were performed changed between 2009 and 2016. In 2009, the most common hospital types were urban nonteaching hospitals (48.4\%), urban teaching hospitals (41.5\%), and rural hospitals (10.1\%) (-Table 7). In 2016, urban teaching hospitals became the most common type (66.6\%) followed by urban nonteaching (26.6\%) and rural (6.8\%). In 2009, the most common hospital size for rTKAs was large (61.3\%) followed by medium (24.8\%) and small (13.9\%). In 2016, the proportion of rTKAs performed at large hospitals decreased (46.4\%), with a concordant slight increase in medium (28.5\%) and small (25.1\%) hospitals.

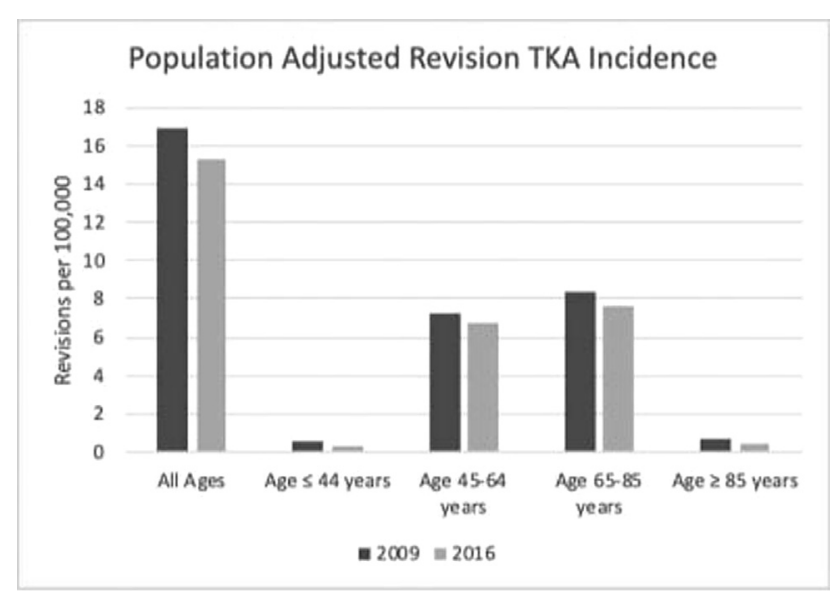

Fig. 3 Population of adjusted incidence of total knee arthroplasty (TKA) revisions in different age groups (dark gray is female, and light gray is male).

\section{Complications, Lengths of Stay, and Discharge Dispositions}

The odds of several complications decreased significantly from 2009 to 2016 (-Table 8). The rate of transfusions decreased significantly from $21.3 \%$ in 2009 to $6.5 \%$ in 2016 (OR, 0.262; 95\% CI, 0.252-0.274; $p<0.001$ ). Other complications that decreased included urinary tract infections (3.5\% in 2009, 2.0\% in 2016; OR, 0.69; 95\% CI, 0.636-0.746; $p<0.001)$, cardiac arrests $(0.13 \%$ in $2009,0.05 \%$ in 2016 ; OR, $0.399 ; 95 \% \mathrm{CI}, 0.232-0.687 ; p=0.001)$, and mortality $(0.19 \%$ in 2009, $0.09 \%$ in 2016; OR, 0.651; 95\% CI, 0.432-0.981; $p=0.040$ ). The incidence of pneumoniae increased significantly from $0.0 \%$ in 2009 to $0.15 \%$ in 2016 (OR, $1.603 \times 10^{10}$; $\left.95 \% \mathrm{CI}, \quad 1.603 \times 10^{10}-1.06 \times 10^{10} ; p<0.001\right)$. Myocardial infarctions also increased between 2009 (3.96\%) and 2016 (4.15\%; OR, 1.129; 95\% CI, 1.058-1.204; $p<0.001$ ). The mean hospital LOS decreased $19.5 \%$ over our study period, from 4.1 days in 2009 to 3.1 days in $2016(p<0.001$; - Table 6). Discharge to an SNF decreased from 31.6\% in 2009 to $26.5 \%$ in 2016 (OR, 0.927; 95\% CI, 0.901-0.954; $p<0.001$ ).

\section{Costs, Charges, and Payer Types}

The trend for both costs and charges of rTKAs increased significantly between 2009 and 2016 (-Table 5). The mean charges increased by $43.4 \%$ ( $\$ 106,956.76$ vs. $\$ 74,575.44$; $p<0.001)$ and the mean costs increased by $18.7 \%$ $(\$ 27,427.02$ vs. $\$ 23,103.07 ; p<0.001)$. Throughout the study period, there were significant changes in the distribution of

Table 3 Population of adjusted incidence of TKA revisions in different age groups (2009-2016)

\begin{tabular}{|l|l|l|l|l|l|l|l|l|l|}
\hline \multicolumn{2}{|l|}{ Population adjusted } \\
\hline TKA revisions & $\mathbf{2 0 0 9}$ & $\mathbf{2 0 1 0}$ & $\mathbf{2 0 1 1}$ & $\mathbf{2 0 1 2}$ & $\mathbf{2 0 1 3}$ & $\mathbf{2 0 1 4}$ & $\mathbf{2 0 1 5}$ & $\mathbf{2 0 1 6}$ & \\
\hline All ages & 16.8416 & $58,431.0000$ & $61,969.0000$ & $58,930.0000$ & $60,315.0000$ & $62,560.0000$ & $50,465.0000$ & 15.2845 & $-9.2 \%$ \\
\hline Age $\leq 44$ y & 0.5508 & $2,099.0000$ & $2,256.0000$ & $1,890.0000$ & $1,935.0000$ & $1,925.0000$ & $1,240.0000$ & 0.3834 & $-30.4 \%$ \\
\hline Age $45-64$ y & 7.2161 & $25,997.0000$ & $27,896.0000$ & $26,445.0000$ & $26,445.0000$ & $27,480.0000$ & $21,575.0000$ & 6.8027 & $-5.7 \%$ \\
\hline Age $65-85$ y & 8.4169 & $28,172.0000$ & $29,606.0000$ & $28,650.0000$ & $29,920.0000$ & $30,990.0000$ & $25,740.0000$ & 7.6268 & $-9.4 \%$ \\
\hline Age $\geq 85$ y & 0.6578 & $2,163.0000$ & $2,211.0000$ & $1,945.0000$ & $2,015.0000$ & $2,165.0000$ & $1,910.0000$ & 0.4716 & $-28.3 \%$ \\
\hline
\end{tabular}

Abbreviation: TKA, total knee arthroplasty. 
Table 4 The 10 most common primary diagnoses leading to TKA revision each year (all age groups)

\begin{tabular}{|c|c|c|c|c|}
\hline \multirow[t]{2}{*}{ Rank } & \multicolumn{2}{|l|}{2009} & \multicolumn{2}{|l|}{2010} \\
\hline & Diagnosis (ICD-9 code) & $N(\%)$ & Diagnosis (ICD-9 code) & $N(\%)$ \\
\hline 1 & $\begin{array}{l}\text { Mechanical loosening of prosthetic } \\
\text { joint ( } 996.41)\end{array}$ & 10,175 (19.7\%) & $\begin{array}{l}\text { Mechanical loosening of prosthetic } \\
\text { joint (996.41) }\end{array}$ & $12,819(21.9 \%)$ \\
\hline 2 & $\begin{array}{l}\text { Infection and inflammatory reaction } \\
\text { due to internal joint prosthesis } \\
(996.66)\end{array}$ & $7,600(14.7 \%)$ & $\begin{array}{l}\text { Other mechanical complication of } \\
\text { prosthetic joint implant (996.47) }\end{array}$ & $11,459(19.6 \%)$ \\
\hline 3 & $\begin{array}{l}\text { Other complications due to internal } \\
\text { joint prosthesis ( } 996.77)\end{array}$ & $6,392(12.4 \%)$ & $\begin{array}{l}\text { Infection and inflammatory reaction } \\
\text { due to internal joint prosthesis } \\
(996.66)\end{array}$ & $8,502(14.6 \%)$ \\
\hline 4 & $\begin{array}{l}\text { Broken prosthetic joint implant } \\
\text { (996.43) }\end{array}$ & $6,325(12.2 \%)$ & $\begin{array}{l}\text { Other complications due to internal } \\
\text { joint prosthesis ( } 996.77)\end{array}$ & 7,437 (12.7\%) \\
\hline 5 & $\begin{array}{l}\text { Other mechanical complication of } \\
\text { prosthetic joint implant (996.47) }\end{array}$ & $4,619(8.9 \%)$ & $\begin{array}{l}\text { Dislocation of prosthetic joint } \\
(996.42)\end{array}$ & $4,110(7.0 \%)$ \\
\hline 6 & $\begin{array}{l}\text { Dislocation of prosthetic joint } \\
(996.42)\end{array}$ & $3,772(7.3 \%)$ & $\begin{array}{l}\text { Other acquired deformities of knee } \\
\text { (736.6) }\end{array}$ & $3,574(6.1 \%)$ \\
\hline 7 & $\begin{array}{l}\text { Other acquired deformities of knee } \\
\text { (736.6) }\end{array}$ & $2,713(5.3 \%)$ & $\begin{array}{l}\text { Articular bearing surface wear of } \\
\text { prosthetic joint (996.46) }\end{array}$ & $1,607(2.8 \%)$ \\
\hline 8 & $\begin{array}{l}\text { Other mechanical complication of } \\
\text { other internal orthopedic device, im- } \\
\text { plant, and graft (996.49) }\end{array}$ & $2,235(4.3 \%)$ & $\begin{array}{l}\text { Other mechanical complication of } \\
\text { other internal orthopedic device, im- } \\
\text { plant, and graft (996.49) }\end{array}$ & $1,425(2.4 \%)$ \\
\hline 9 & $\begin{array}{l}\text { Articular bearing surface wear of } \\
\text { prosthetic joint }(996.46)\end{array}$ & $1,810(3.5 \%)$ & $\begin{array}{l}\text { Broken prosthetic joint implant } \\
(996.43)\end{array}$ & $733(1.3 \%)$ \\
\hline 10 & Peri-prosthetic osteolysis (996.45) & $585(1.1 \%)$ & $\begin{array}{l}\text { Osteoarthrosis, localized, not speci- } \\
\text { fied whether primary or secondary, } \\
\text { lower leg (715.36) }\end{array}$ & $676(1.2 \%)$ \\
\hline \multirow[t]{2}{*}{ Rank } & \multicolumn{2}{|l|}{2011} & \multicolumn{2}{|l|}{2012} \\
\hline & Diagnosis (ICD-9 code) & $N(\%)$ & Diagnosis (ICD-9 code) & $N(\%)$ \\
\hline 1 & $\begin{array}{l}\text { Mechanical loosening of prosthetic } \\
\text { joint }(996.41)\end{array}$ & 12,948 (20.9\%) & $\begin{array}{l}\text { Mechanical loosening of prosthetic } \\
\text { joint }(996.41)\end{array}$ & $12,290(20.9 \%)$ \\
\hline 2 & $\begin{array}{l}\text { Other mechanical complication of } \\
\text { prosthetic joint implant (996.47) }\end{array}$ & 12,096 (19.5\%) & $\begin{array}{l}\text { Other mechanical complication of } \\
\text { prosthetic joint implant (996.47) }\end{array}$ & $11,800(20.0 \%)$ \\
\hline 3 & $\begin{array}{l}\text { Infection and inflammatory reaction } \\
\text { due to internal joint prosthesis } \\
(996.66)\end{array}$ & $8,618(13.9 \%)$ & $\begin{array}{l}\text { Infection and inflammatory reaction } \\
\text { due to internal joint prosthesis } \\
(996.66)\end{array}$ & $8,850(15.0 \%)$ \\
\hline 4 & $\begin{array}{l}\text { Other complications due to internal } \\
\text { joint prosthesis (996.77) }\end{array}$ & $7,268(11.7 \%)$ & $\begin{array}{l}\text { Other complications due to internal } \\
\text { joint prosthesis (996.77) }\end{array}$ & $6,880(11.7 \%)$ \\
\hline 5 & $\begin{array}{l}\text { Dislocation of prosthetic joint } \\
(996.42)\end{array}$ & $4,834(7.8 \%)$ & $\begin{array}{l}\text { Dislocation of prosthetic joint } \\
(996.42)\end{array}$ & 5,035 (8.5\%) \\
\hline 6 & $\begin{array}{l}\text { Other acquired deformities of knee } \\
\text { (736.6) }\end{array}$ & $3,913(6.3 \%)$ & $\begin{array}{l}\text { Other acquired deformities of knee } \\
\text { (736.6) }\end{array}$ & 2,255 (3.8\%) \\
\hline 7 & $\begin{array}{l}\text { Other mechanical complication of } \\
\text { other internal orthopedic device, im- } \\
\text { plant, and graft (996.49) }\end{array}$ & 1,795 (2.9\%) & $\begin{array}{l}\text { Articular bearing surface wear of } \\
\text { prosthetic joint }(996.46)\end{array}$ & 1,535 (2.6\%) \\
\hline 8 & $\begin{array}{l}\text { Articular bearing surface wear of } \\
\text { prosthetic joint }(996.46)\end{array}$ & 1,668 (2.7\%) & $\begin{array}{l}\text { Aftercare following explantation of } \\
\text { joint prosthesis (V54.82) }\end{array}$ & $1,440(2.4 \%)$ \\
\hline 9 & $\begin{array}{l}\text { Broken prosthetic joint implant } \\
(996.43)\end{array}$ & $828(1.3 \%)$ & $\begin{array}{l}\text { Other mechanical complication of } \\
\text { other internal orthopedic device, im- } \\
\text { plant, and graft (996.49) }\end{array}$ & 1,315 (2.2\%) \\
\hline 10 & $\begin{array}{l}\text { Infection and inflammatory reaction } \\
\text { due to other internal orthopedic de- } \\
\text { vice, implant, and graft (996.67) }\end{array}$ & $680(1.1 \%)$ & $\begin{array}{l}\text { Osteoarthrosis, localized, not speci- } \\
\text { fied whether primary or secondary, } \\
\text { lower leg (715.36) }\end{array}$ & 720 (1.2\%) \\
\hline
\end{tabular}


Table 4 (Continued)

\begin{tabular}{|c|c|c|c|c|}
\hline \multirow[t]{2}{*}{ Rank } & \multicolumn{2}{|l|}{2013} & \multicolumn{2}{|l|}{2014} \\
\hline & Diagnosis (ICD-9 code) & $N(\%)$ & Diagnosis (ICD-9 code) & $N(\%)$ \\
\hline 1 & $\begin{array}{l}\text { Mechanical loosening of prosthetic } \\
\text { joint }(996.41)\end{array}$ & $12,660(21.0 \%)$ & $\begin{array}{l}\text { Mechanical loosening of prosthetic } \\
\text { joint }(996.41)\end{array}$ & $12,810(20.5 \%)$ \\
\hline 2 & $\begin{array}{l}\text { Other mechanical complication of } \\
\text { prosthetic joint implant (996.47) }\end{array}$ & $12,135(20.1 \%)$ & $\begin{array}{l}\text { Other mechanical complication of } \\
\text { prosthetic joint implant (996.47) }\end{array}$ & $12,285(19.6 \%)$ \\
\hline 3 & $\begin{array}{l}\text { Infection and inflammatory reaction } \\
\text { due to internal joint prosthesis } \\
(996.66)\end{array}$ & 9,565 (15.9\%) & $\begin{array}{l}\text { Infection and inflammatory reaction } \\
\text { due to internal joint prosthesis } \\
(996.66)\end{array}$ & $10,195(16.3 \%)$ \\
\hline 4 & $\begin{array}{l}\text { Other complications due to internal } \\
\text { joint prosthesis (996.77) }\end{array}$ & $6,695(11.1 \%)$ & $\begin{array}{l}\text { Other complications due to internal } \\
\text { joint prosthesis (996.77) }\end{array}$ & $6,925(11.1 \%)$ \\
\hline 5 & $\begin{array}{l}\text { Dislocation of prosthetic joint } \\
(996.42)\end{array}$ & $4,650(7.7 \%)$ & $\begin{array}{l}\text { Dislocation of prosthetic joint } \\
(996.42)\end{array}$ & $5,090(8.1 \%)$ \\
\hline 6 & $\begin{array}{l}\text { Other acquired deformities of knee } \\
\text { (736.6) }\end{array}$ & $2,240(3.7 \%)$ & $\begin{array}{l}\text { Other acquired deformities of knee } \\
\text { (736.6) }\end{array}$ & $2,040(3.3 \%)$ \\
\hline 7 & $\begin{array}{l}\text { Aftercare following explantation of } \\
\text { joint prosthesis (V54.82) }\end{array}$ & $1,695(2.8 \%)$ & $\begin{array}{l}\text { Aftercare following explantation of } \\
\text { joint prosthesis (V54.82) }\end{array}$ & $1,865(3.0 \%)$ \\
\hline 8 & $\begin{array}{l}\text { Articular bearing surface wear of } \\
\text { prosthetic joint (996.46) }\end{array}$ & $1,375(2.3)$ & $\begin{array}{l}\text { Other mechanical complication of } \\
\text { other internal orthopedic device, im- } \\
\text { plant, and graft (996.49) }\end{array}$ & $1,625(2.6 \%)$ \\
\hline 9 & $\begin{array}{l}\text { Other mechanical complication of } \\
\text { other internal orthopedic device, im- } \\
\text { plant, and graft (996.49) }\end{array}$ & $1,330(2.2 \%)$ & $\begin{array}{l}\text { Articular bearing surface wear of } \\
\text { prosthetic joint }(996.46)\end{array}$ & $1,345(2.1 \%)$ \\
\hline 10 & $\begin{array}{l}\text { Osteoarthrosis, localized, not speci- } \\
\text { fied whether primary or secondary, } \\
\text { lower leg }(715.36)\end{array}$ & $745(1.2 \%)$ & $\begin{array}{l}\text { Peri-prosthetic fracture around pros- } \\
\text { thetic joint (996.44) }\end{array}$ & $725(1.2 \%)$ \\
\hline \multirow[t]{2}{*}{ Rank } & \multicolumn{2}{|l|}{2015} & \multicolumn{2}{|l|}{2015} \\
\hline & Diagnosis (ICD-9 code) & $N(\%)$ & Diagnosis (ICD-10 code) & $N(\%)$ \\
\hline 1 & $\begin{array}{l}\text { Other mechanical complication of } \\
\text { prosthetic joint implant (996.47) }\end{array}$ & $9,800(15.3 \%)$ & $\begin{array}{l}\text { Infection and inflammatory reaction } \\
\text { due to internal right knee prosthesis - } \\
\text { initial encounter (T84.53XA) }\end{array}$ & $1,990(3.1 \%)$ \\
\hline 2 & $\begin{array}{l}\text { Mechanical loosening of prosthetic } \\
\text { joint }(996.41)\end{array}$ & $9,490(14.8 \%)$ & $\begin{array}{l}\text { Infection and inflammatory reaction } \\
\text { due to internal left knee prosthesis - } \\
\text { initial encounter (T84.54XA) }\end{array}$ & $1,975(3.1 \%)$ \\
\hline 3 & $\begin{array}{l}\text { Infection and inflammatory reaction } \\
\text { due to internal joint prosthesis } \\
(996.66)\end{array}$ & $8,300(13.0 \%)$ & $\begin{array}{l}\text { Mechanical loosening of internal right } \\
\text { knee prosthetic joint - initial en- } \\
\text { counter (T84.032A) }\end{array}$ & $645(1.0 \%)$ \\
\hline 4 & $\begin{array}{l}\text { Other complications due to internal } \\
\text { joint prosthesis (996.77) }\end{array}$ & $4,855(7.6 \%)$ & $\begin{array}{l}\text { Pain due to internal orthopedic pros- } \\
\text { thetic devices, implants and grafts - } \\
\text { initial encounter (T84.84XA) }\end{array}$ & $625(1.0 \%)$ \\
\hline 5 & $\begin{array}{l}\text { Dislocation of prosthetic joint } \\
(996.42)\end{array}$ & 3,585 (5.6\%) & $\begin{array}{l}\text { Mechanical loosening of internal left } \\
\text { knee prosthetic joint - initial en- } \\
\text { counter (T84.033A) }\end{array}$ & $550(0.9 \%)$ \\
\hline 6 & $\begin{array}{l}\text { Aftercare following explantation of } \\
\text { joint prosthesis (V54.82) }\end{array}$ & $1,545(2.4 \%)$ & $\begin{array}{l}\text { Instability of internal right knee } \\
\text { prosthesis - initial encounter } \\
\text { (T84.022A) }\end{array}$ & $490(0.8 \%)$ \\
\hline 7 & $\begin{array}{l}\text { Other acquired deformities of knee } \\
\text { (736.6) }\end{array}$ & $1,345(2.1 \%)$ & $\begin{array}{l}\text { Instability of internal left knee pros- } \\
\text { thesis - initial encounter (T84.023A) }\end{array}$ & $460(0.7 \%)$ \\
\hline 8 & $\begin{array}{l}\text { Other mechanical complication of } \\
\text { other internal orthopedic device, im- } \\
\text { plant, and graft (996.49) }\end{array}$ & $1,175(1.8 \%)$ & $\begin{array}{l}\text { Other mechanical complication of in- } \\
\text { ternal right knee prosthesis, initial } \\
\text { encounter (T84.092A) }\end{array}$ & $385(0.6 \%)$ \\
\hline 9 & $\begin{array}{l}\text { Articular bearing surface wear of } \\
\text { prosthetic joint }(996.46)\end{array}$ & $1,020(1.6 \%)$ & $\begin{array}{l}\text { Other mechanical complication of in- } \\
\text { ternal left knee prosthesis - initial } \\
\text { encounter (T84.093A) }\end{array}$ & $370(0.6 \%)$ \\
\hline
\end{tabular}


Table 4 (Continued)

\begin{tabular}{|c|c|c|c|c|}
\hline 10 & $\begin{array}{l}\text { Peri-prosthetic fracture around pros- } \\
\text { thetic joint }(996.44)\end{array}$ & $705(1.1 \%)$ & $\begin{array}{l}\text { Displaced bicondylar fracture of left } \\
\text { tibia - initial encounter for closed } \\
\text { fracture (S82.142A) }\end{array}$ & $335(0.5 \%)$ \\
\hline \multirow[t]{2}{*}{ Rank } & \multicolumn{2}{|l|}{2016} & & \\
\hline & Diagnosis (ICD-10 code) & $N(\%)$ & & \\
\hline 1 & $\begin{array}{l}\text { Infection and inflammatory reaction } \\
\text { due to internal right knee prosthesis - } \\
\text { initial encounter (T84.53XA) }\end{array}$ & $8,440(13.0 \%)$ & & \\
\hline 2 & $\begin{array}{l}\text { Infection and inflammatory reaction } \\
\text { due to internal left knee prosthesis - } \\
\text { initial encounter (T84.54XA) }\end{array}$ & $7,990(12.3 \%)$ & & \\
\hline 3 & $\begin{array}{l}\text { Mechanical loosening of internal right } \\
\text { knee prosthetic joint - initial en- } \\
\text { counter (T84.032A) }\end{array}$ & $2,735(4.2 \%)$ & & \\
\hline 4 & $\begin{array}{l}\text { Pain due to internal orthopedic pros- } \\
\text { thetic devices, implants and grafts - } \\
\text { initial encounter (T84.84XA) }\end{array}$ & $2,630(4.0 \%)$ & & \\
\hline 5 & $\begin{array}{l}\text { Mechanical loosening of internal left } \\
\text { knee prosthetic joint - initial en- } \\
\text { counter (T84.033A) }\end{array}$ & $2,570(4.0 \%)$ & & \\
\hline 6 & $\begin{array}{l}\text { Instability of internal left knee pros- } \\
\text { thesis - initial encounter (T84.023A) }\end{array}$ & $2,050(3.2 \%)$ & & \\
\hline 7 & $\begin{array}{l}\text { Instability of internal right knee } \\
\text { prosthesis - initial encounter } \\
(\mathrm{T} 84.022 \mathrm{~A})\end{array}$ & $2,045(3.1 \%)$ & & \\
\hline 8 & $\begin{array}{l}\text { Displaced bicondylar fracture of right } \\
\text { tibia - initial encounter for closed } \\
\text { fracture (S82.141A) }\end{array}$ & $1,245(1.9 \%)$ & & \\
\hline 9 & $\begin{array}{l}\text { Displaced bicondylar fracture of left } \\
\text { tibia - initial encounter for closed } \\
\text { fracture (S82.142A) }\end{array}$ & $1,240(1.9 \%)$ & & \\
\hline 10 & $\begin{array}{l}\text { Other mechanical complication of in- } \\
\text { ternal left knee prosthesis - initial } \\
\text { encounter (T84.093A) }\end{array}$ & $1,035(1.6 \%)$ & & \\
\hline
\end{tabular}

Abbreviations: ICD, International Classification of Diseases; TKA, total knee arthroplasty.

the most common payer type $(p<0.001)$. There was an increase in the number of rTKAs cases that were covered by Medicare ( 57.5 vs. $56.6 \%$ ) and Medicaid (5.4 vs. $3.7 \%$ ). Private insurance ( 31.8 vs. $33.3 \%$ ), self-payers ( 0.4 vs. $0.7 \%$ ), and other types of payers (4.8 vs. $5.7 \%$ ) covered a fewer number of rTKAs ( - Table 9 ).

\section{Discussion}

The incidence of primary TKAs is on the rise in the United States and is expected to increase $673 \%$ between 2005 and 2030, to an estimated 3.48 million procedures per year. ${ }^{5}$ Additionally, hospital charges for rTKAs were projected to increase $450 \%$ over a 10 -year period to $\$ 4.1$ billion in $2015 .^{13}$ In our study, we found that the incidence of rTKAs decreased $4.3 \%$ between 2009 and 2016. It is possible that improvements in surgical techniques and perioperative care, as well as advancements in implant designs have contributed to this apparent decrease in rTKA procedures. However, there remains an increased economic burden on patients, hospitals, and the health care system. The mean charges have increased to $\$ 106,956.76$ and the mean costs have risen to $\$ 27,427.02$. Our study found that Medicare was the primary payer between 2009 and 2016. This may have a substantial economic burden on hospitals and the health care system because it has been suggested that Medicare reimbursements are only 32 to $38 \%$ of charges. ${ }^{13,20}$

Our study has some limitations. Due to its retrospective nature, the inherent shortcomings associated with this study design must be noted. These included the potential for selection bias, classification bias, or information bias. Another limitation of this study is the challenge faced when comparing results before and after the transition from ICD-9 to ICD-10 codes. In addition, the data were taken from an inpatient database, so subsequent readmissions and those associated costs may not have been captured. Unfortunately, the data cannot be utilized to discuss 90day or episode-of-care costs. ICD-9 codes do not account for 


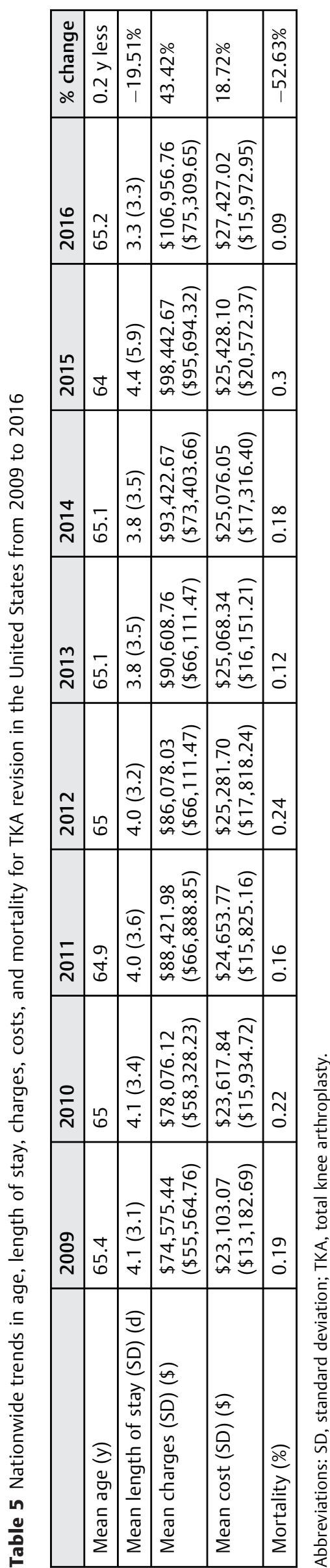

Table 6 Patient demographics

\begin{tabular}{|c|c|c|c|}
\hline & 2009 & 2016 & $p$-Value \\
\hline $\begin{array}{l}\text { Mean age } \\
(\mathrm{SD})(\mathrm{y})\end{array}$ & $65.4(11.5)$ & $65.2(10.7)$ & $<0.001$ \\
\hline \multicolumn{4}{|l|}{ Sex } \\
\hline Male & $21,710(42.1 \%)$ & $20,565(41.6 \%)$ & \multirow[t]{2}{*}{0.144} \\
\hline Female & $29,872(57.9 \%)$ & $28,830(58.4 \%)$ & \\
\hline $\begin{array}{l}\text { Mean length of } \\
\text { stay (SD) (d) }\end{array}$ & $4.1(3.5)$ & $3.3(3.3)$ & $<0.001$ \\
\hline \multicolumn{4}{|c|}{ Severity of illness score $(N[\%])$} \\
\hline Minor & $8,676(16.8 \%)$ & $16,235(32.8 \%)$ & \multirow[t]{4}{*}{$<0.001$} \\
\hline Moderate & $34,561(66.9 \%)$ & $26,850(54.3 \%)$ & \\
\hline Major & $7,411(14.3 \%)$ & $5,610(11.3 \%)$ & \\
\hline Extreme & $1,022(2.0 \%)$ & $735(1.5 \%)$ & \\
\hline
\end{tabular}

Abbreviation: SD, standard deviation.

Table 7 Most common hospital type and size used for TKA revisions (2009 and 2016)

\begin{tabular}{|c|c|c|c|}
\hline & 2009 & 2016 & $p$-Value \\
\hline \multicolumn{4}{|c|}{ Hospital type (N [\%]) } \\
\hline Rural & $5,110(10.1 \%)$ & $3,350(6.8 \%)$ & \multirow[t]{3}{*}{$<0.001$} \\
\hline $\begin{array}{l}\text { Urban, } \\
\text { nonteaching }\end{array}$ & $24,502(48.4 \%)$ & $13,145(26.6 \%)$ & \\
\hline $\begin{array}{l}\text { Urban, } \\
\text { teaching }\end{array}$ & 20,989 (41.5\%) & $32,935(66.6 \%)$ & \\
\hline \multicolumn{4}{|c|}{ Hospital bed size (N [\%]) } \\
\hline Small & $7,019(13.9 \%)$ & $12,420(25.1 \%)$ & \multirow[t]{3}{*}{$<0.001$} \\
\hline Medium & $12,550(24.8 \%)$ & $14,095(28.5 \%)$ & \\
\hline Large & $31,032(61.3 \%)$ & $22,915(46.4 \%)$ & \\
\hline
\end{tabular}

Abbreviation: TKA, total knee arthroplasty.

laterality. Therefore, there was a degree of uncertainty regarding whether or not the complications were specific to the operative side. The data were also derived from a large database, and as such may not be entirely accurate due to the possibility of missing or incorrect patient data. However, since the NIS database provides such a large sample size by covering roughly $20 \%$ of all U.S. community hospitals, it is still considered to be an accurate representation of all hospital admissions, procedures, diagnoses, and discharges within the United States. ${ }^{17}$

This study analyzed the economics of rTKAs, evaluating the costs, charges, and payer types associated with the procedure. Delanois et al used the HCUP NIS database to study 337,597 rTKAs between 2009 and 2013. ${ }^{1}$ They determined that charges increased to a mean of $\$ 75,028.07$ and that Medicare was the primary payer for most of the rTKAs performed. ${ }^{1}$ Similarly, our study found an increase in mean total charge $(\$ 106,956.76$ vs. $\$ 74,575.44 ; p<0.001)$ and that Medicare was the primary payer between 2009 and 2016. Delanois et al also found the mean LOS to be 4.5 days and 
Table 8 Multivariate logistic regression for complications of TKA revisions (2009 compared with 2016)

\begin{tabular}{|l|l|l|l|l|}
\hline Complication & $\begin{array}{l}\mathbf{2 0 0 9} \\
(\boldsymbol{n}=\mathbf{5 1 , 6 7 0 )}\end{array}$ & $\begin{array}{l}\mathbf{2 0 1 6} \\
(\boldsymbol{n}=\mathbf{4 9 , 4 3 0 )}\end{array}$ & OR (95\% Cl) & $p$-Value \\
\hline Myocardial infarction & $2,045(4.0 \%)$ & $2,050(4.1 \%)$ & $1.129(1.058-1.204)$ & $<0.001$ \\
\hline $\begin{array}{l}\text { Pulmonary embolism/deep } \\
\text { vein thrombosis }\end{array}$ & $551(1.1 \%)$ & $350(0.7 \%)$ & $0.895(0.776-1.031)$ & 0.125 \\
\hline Cardiac arrest & $68(0.1 \%)$ & $25(0.1 \%)$ & $0.399(0.232-0.687)$ & 0.001 \\
\hline Transfusion & $11,014(21.3 \%)$ & $3,200(6.5 \%)$ & $0.262(0.252-0.274)$ & $<0.001$ \\
\hline Pneumonia & $0(0.0 \%)$ & $75(0.2 \%)$ & $1.603 \times 10^{10}\left(1.06 \times 10^{10}-1.063 \times 10^{10}\right)$ & - \\
\hline Urinary tract infection & $1,790(3.5 \%)$ & $995(2.0 \%)$ & $0.690(0.636-0.746)$ & $<0.001$ \\
\hline Discharge to skilled nursing facility & $16,351(31.6 \%)$ & $13,100(26.5)$ & $0.927(0.901-0.954)$ & $<0.001$ \\
\hline Death & $96(0.2 \%)$ & $45(0.1 \%)$ & $0.651(0.432-0.981)$ & 0.040 \\
\hline
\end{tabular}

Abbreviations: $\mathrm{Cl}$, confidence interval; OR, odds ratio; TKA, total knee arthroplasty.

Table 9 Charges, costs, and payer types associated with TKA revision

\begin{tabular}{|c|c|c|c|}
\hline Mean charges (SD) (\$) & $\$ 74,575.44(\$ 55,564.76)$ & $\$ 106,956.76(\$ 75,309.65)$ & $<0.001$ \\
\hline Mean cost (SD) (\$) & $\$ 23,103.07(\$ 13,182.69)$ & $\$ 27,427.02(\$ 15,972.95)$ & $<0.001$ \\
\hline \multicolumn{4}{|l|}{ Payer type (N [\%]) } \\
\hline Medicare & $29,160(56.6 \%)$ & $28,420(57.5 \%)$ & \multirow[t]{5}{*}{$<0.001$} \\
\hline Medicaid & $1,893(3.7 \%)$ & $2,655(5.4 \%)$ & \\
\hline Private insurance & $17,146(33.3 \%)$ & $15,705(31.8 \%)$ & \\
\hline Self-pay & $365(0.7 \%)$ & $195(0.4 \%)$ & \\
\hline Other & 2,937 (5.7\%) & $2,390(4.8 \%)$ & \\
\hline
\end{tabular}

Abbreviations: SD, standard deviation; TKA, total knee arthroplasty.

determined a downward trend in LOS between 2009 and $2013 .^{1}$ This is concordant with our finding that the LOS is decreasing (3.3 vs. 4.1 days; $p<0.001$ ).

Bozic et al also utilized the HCUP NIS database to analyze 60,355 rTKAs performed between 2005 and 2006. ${ }^{21}$ They found that the economic burden of rTKAs remained large and that most rTKAs were performed in large urban nonteaching hospitals and on Medicare patients. ${ }^{21}$ Similarly, our study found Medicare to be the most common payer type for rTKAs. However, we found that the charges and LOS have changed significantly over the past 10 years. Bozic et al reported that the mean total charges were $\$ 49,360$ and the LOS to be 5.1 days. ${ }^{21}$ Our study found that by 2016 charges increased to $\$ 106,956.76$ and LOS decreased to 3.3 days. Bozic et al studied data from 2005 to 2006, which can contribute to the differences in the findings of these studies. ${ }^{21}$ However, the trend of increasing charges despite decreasing LOS is seen in both studies.

The trend of decreased LOS for rTKAs is corroborated by several studies. ${ }^{4,14,15}$ Cram et al analyzed 318,563 patients who underwent rTKAs between 1991 and 2010. ${ }^{14}$ They found that LOS decreased from 8.9 days in 1991 to 5 days in $2010 .{ }^{14}$ Our study identified a similar downward trend, with LOS decreasing from 4.1 to 3.3 days between 2009 and 2016. Our study also found that the use of blood transfusions decreased, which is a noted trend within the literature. ${ }^{15,22}$ Husted et al analyzed 712 patients who received either a total hip arthroplasty (THA) or a TKA between 2003 and 2005 and found that decreased blood transfusions was correlated with decreased LOS. ${ }^{15}$ Similarly, in our study, blood transfusions decreased from 11,014 to 3,200 and LOS also decreased from 4.1 to 3.3 days.

The literature shows that increased LOS can contribute to a patient's need to be discharged to an SNF. ${ }^{23}$ Ramkumar et al analyzed 3,218,419 TKA patients between 2009 and 2013, and 403,575 of them were sent to an SNF. ${ }^{23}$ The study found that increased LOS was one of the key risk factors that led to a patient being sent to an SNF. ${ }^{23}$ Our study found the number of patients discharged to an SNF decreased from 31.6 to $26.5 \%$ and LOS decreased from 4.1 to 3.3 days. Therefore, that study can give reason to believe that there is an association between LOS and SNF.

The main causes of TKA failure that required revision surgery within our study population are comparable with the most common reasons found in the literature, including: loosenings, infections, painful reasons, osteolyses, and instabilities. ${ }^{1,6-10}$ Dalury et al conducted a retrospective analysis of 820 cases from 2000 to 2012 to determine the reasons for rTKAs. ${ }^{7}$ They found that the most common causes for rTKAs were aseptic loosening, infection, polyethylene wear, 
instability, pain/stiffness, osteolysis, and malposition/malalignment. ${ }^{7}$ Both studies identify the same top causes of rTKAs, however, with varying ranking and incidence volume. The discrepancies can be due to the differing time frames in which the studies were performed. Our study demonstrated that the causes for rTKAs have changed over time, supporting this explanation.

The current literature shows that cases of primary TKAs and rTKAs are increasing. ${ }^{2-5}$ However, our study found that overall rTKAs decreased by $4.34 \%$ (9.25\% when population adjusted) between 2009 and 2016. Despite the continued increase in amount of primary TKAs, the rate was not accelerated after 2007.2,3 Therefore, this slowed growth could explain a slight dip in overall rTKAs in our data from 2009 to 2016. Within our study, more women received rTKAs than men. Bayliss et al analyzed rates of primary and revision THAs and TKAs for men and women from 1991 to 2011 . $^{24}$ They found that more women received TKAs, while more men received rTKAs. ${ }^{24}$ This contrasts our study's findings that women had increased cases of TKAs and rTKAs compared with men. The difference between our studies can be due to the differences in the population sets (United States vs. United Kingdom).

The literature shows that LOS is a key factor of medical costs. ${ }^{11,12}$ Lovald et al created a study using the Medicare 5\% Limited Data Set to gather data of patients who had a TKA between 1997 and 2009. ${ }^{12}$ The patients were then further stratified by the amount of LOS they had. At the end of 2 years, they found patients with lower LOS had decreased medical costs. ${ }^{12}$ This is in contrast with our study, where we found that even though our LOS had decreased, the costs still increased.

\section{Conclusion}

This study provides a better understanding regarding the burden of TKA revisions in the United States. There was an overall decrease in rTKAs among all age groups by $4.34 \%$ (9.25\% when population adjusted). Aseptic revisions became increasingly prevalent as septic rTKA rates decreased. Positive trends were noted, such as decreased mean LOS, discharges to SNF, and rates of blood transfusion. This may be due to an increase in rTKA procedures being performed at urban teaching hospitals. Still, the cost and charges increased significantly between 2009 and 2016. The economic burden of rTKAs remains large, and we must continue to improve patient outcomes in this population. This includes noting longer term trends for rTKAs with current ICD codes and continuing to improve implant reliability and survivorship. Future research should analyze how the economic burden is trending, evaluate readmission rates for patients who underwent a primary and/or rTKA, and determine whether treatment prior to TKA can help decrease overall cost and economic burden of rTKA on the patient, hospital, and health care system. This report can be used to help educate medical providers to understand, prevent, and educate their patients on the outcomes that may result from a primary and/or revised TKAs.

\section{Conflict of Interest}

G.R.S. reports personal fees from Acelity, personal fees from Biomet, personal fees from Convatec, other from Force Therapeutics, personal fees from Medtronic, other from Operation Walk USA, grants and personal fees from Pacira, personal fees from Zimmer, outside the submitted work. R.E.D. reports grants from Flexion Therapeutics, grants from Orthofix, grants from Stryker, grants from TissueGene, grants from United Orthopedics, outside the submitted work. M.A.M. reports personal fees from Cymedica, personal fees from Flexion Therapeutics, grants and personal fees from Johnson \& Johnson, other from Peerwell, personal fees from Pfizer, grants, personal fees, and other from Stryker, grants and personal fees from TissueGene, other from USMI, outside the submitted work. All the other authors report no conflict of interest.

\section{References}

1 Delanois RE, Mistry JB, Gwam CU, Mohamed NS, Choksi US, Mont MA. Current epidemiology of revision total knee arthroplasty in the United States. J Arthroplasty 2017;32(09):2663-2668

2 Inacio MCS, Paxton EW, Graves SE, Namba RS, Nemes S. Projected increase in total knee arthroplasty in the United States - an alternative projection model. Osteoarthritis Cartilage 2017;25 (11):1797-1803

3 Sloan M, Premkumar A, Sheth NP. Projected volume of primary total joint arthroplasty in the U.S., 2014 to 2030. J Bone Joint Surg Am 2018;100(17):1455-1460

4 Bozic KJ, Kamath AF, Ong K, et al. Comparative epidemiology of revision arthroplasty: failed THA poses greater clinical and economic burdens than failed TKA. Clin Orthop Relat Res 2015;473 (06):2131-2138

5 Kurtz S, Ong K, Lau E, Mowat F, Halpern M. Projections of primary and revision hip and knee arthroplasty in the United States from 2005 to 2030. J Bone Joint Surg Am 2007;89(04):780-785

6 Calliess T, Ettinger M, Hülsmann N, Ostermeier S, Windhagen $\mathrm{H}$. Update on the etiology of revision TKA - evident trends in a retrospective survey of 1449 cases. Knee 2015;22(03):174-179

7 Dalury DF, Pomeroy DL, Gorab RS, Adams MJ. Why are total knee arthroplasties being revised? J Arthroplasty 2013;28(8, Suppl):120-121

8 Schroer WC, Berend KR, Lombardi AV, et al. Why are total knees failing today? Etiology of total knee revision in 2010 and 2011. J Arthroplasty 2013;28(8, Suppl):116-119

9 Gonzalez MH, Mekhail AO. The failed total knee arthroplasty: evaluation and etiology. J Am Acad Orthop Surg 2004;12(06): 436-446

10 Sharkey PF, Lichstein PM, Shen C, Tokarski AT, Parvizi J. Why are total knee arthroplasties failing today-has anything changed after 10 years? J Arthroplasty 2014;29(09):1774-1778

11 Culler SD, Jevsevar DS, Shea KG, Wright KK, Simon AW. The incremental hospital cost and length-of-stay associated with treating adverse events among Medicare beneficiaries undergoing TKA. J Arthroplasty 2015;30(01):19-25

12 Lovald ST, Ong KL, Malkani AL, et al. Complications, mortality, and costs for outpatient and short-stay total knee arthroplasty patients in comparison to standard-stay patients. J Arthroplasty 2014;29(03):510-515

13 Kurtz SM, Ong KL, Schmier J, et al. Future clinical and economic impact of revision total hip and knee arthroplasty. J Bone Joint Surg Am 2007;89(Suppl 3):144-151

14 Cram P, Lu X, Kates SL, Singh JA, Li Y, Wolf BR. Total knee arthroplasty volume, utilization, and outcomes among Medicare beneficiaries, 1991-2010. JAMA 2012;308(12):1227-1236 
15 Husted H, Holm G, Jacobsen S. Predictors of length of stay and patient satisfaction after hip and knee replacement surgery: fasttrack experience in 712 patients. Acta Orthop 2008;79(02):168-173

16 Kim L, Kim J-A, Kim S. A guide for the utilization of Health Insurance Review and Assessment Service National Patient Samples. Epidemiol Health 2014;36(Jul):e2014008

17 HCUP National Inpatient Sample (NIS) Healthcare Cost and Utilization Project (HCUP) 2012. Agency for Healthcare Research and Quality, Rockville, MD. Available at: www.hcup-us.ahrq.gov/nisoverview.jsp

18 Horn SD, Horn RA, Sharkey PD. The Severity of Illness Index as a severity adjustment to diagnosis-related groups. Health Care Financ Rev 1984Suppl(Suppl):36:33-45

19 HCUP Cost-to-Charge Ratio (CCR) for the National Inpatient Sample (NIS). Healthcare Cost and Utilization Project (HCUP) 2017. Agency for Healthcare Research and Quality, Rockville, MD. Available at: www.hcup-us.ahrq.gov/db/ccr/ip-ccr/ip-ccr.jsp
20 Ong KL, Mowat FS, Chan N, Lau E, Halpern MT, Kurtz SM. Economic burden of revision hip and knee arthroplasty in Medicare enrollees. Clin Orthop Relat Res 2006;446:22-28

21 Bozic KJ, Kurtz SM, Lau E, et al. The epidemiology of revision total knee arthroplasty in the United States. Clin Orthop Relat Res 2010;468(01):45-51

22 Bedard NA, Pugely AJ, Lux NR, Liu SS, Gao Y, Callaghan JJ. Recent trends in blood utilization after primary hip and knee arthroplasty. J Arthroplasty 2017;32(03):724-727

23 Ramkumar PN, Gwam C, Navarro SM, et al. Discharge to the skilled nursing facility: patient risk factors and perioperative outcomes after total knee arthroplasty. Ann Transl Med 2019;7 (04):65

24 Bayliss LE, Culliford D, Monk AP, et al. The effect of patient age at intervention on risk of implant revision after total replacement of the hip or knee: a population-based cohort study. Lancet 2017; 389(10077):1424-1430 